\title{
A PRIMITIVE RING WHICH IS A SUM OF TWO WEDDERBURN RADICAL SUBRINGS
}

\author{
A. V. KELAREV
}

(Communicated by Ken Goodearl)

\begin{abstract}
We give an example of a primitive ring which is a sum of two Wedderburn radical subrings. This answers an open question and simplifies the proof of the known theorem that there exists a ring which is not nil but is a sum of two locally nilpotent subrings.
\end{abstract}

Kegel [3] proved that a ring is nilpotent if it is a sum of two nilpotent subrings. The question of whether every ring must be nil if it is the sum of two nil or locally nilpotent subrings was asked in [4] and was considered by several authors. Herstein and Small proved that a PI-ring is locally nilpotent if it is a sum of two nil subrings $([2$, Theorem 2]). There was a conjecture that every ring is locally nilpotent if it is a sum of two locally nilpotent subrings (it is mentioned on p. 775 of [2]). Ferrero and Puczyłowski [1] proved, in particular, that every ring must be locally nilpotent if it is a sum of a right or left $T$-nilpotent subring and a locally nilpotent subring.

The interest in the question increased after Ferrero and Puczyłowski [1] had shown that the famous Koethe problem is equivalent to the fact that every ring is nil if it is a sum of a nil subring and a nilpotent subring. In the survey [6] Puczyłowski suggested that an example be sought of a ring which is not nil but is a sum of two nil subrings.

Indeed, in [5] the author constructed an example of a ring which is not nil but is a sum of two locally nilpotent subrings. A few years later a family of such examples using the semigroup of all partial translations was given by Salwa [7].

However, there is another related question which still remains open. Namely, Puczyłowski [6] asked whether a ring, which is a sum of two Wedderburn radical subrings, must be Baer radical. A ring is said to be Wedderburn radical if it is equal to the sum of its nilpotent ideals. Is is written in $[6$, p. 224] that the answer seems to be 'yes'.

The aim of the present note is twofold. First, we show that there exists a primitive (and so prime) ring which is a sum of two Wedderburn radical subrings. Thus, surprisingly, the answer to the open question above is in fact 'no'. Secondly, we seriously simplify the proof of the main theorem of [5] which answered the first long-standing question above.

Received by the editors July 16, 1996.

1991 Mathematics Subject Classification. Primary 16N40; Secondary 16N60.

Key words and phrases. Nilpotent rings, locally nilpotent rings, nil rings.

The author was supported by a grant of the Australian Research Council. 
Theorem 1. There exists a primitive ring which is a sum of two Wedderburn radical subrings.

Proof. Following [5], let $S$ be the free semigroup with two generators $a$ and $b$. For $s \in S$, let $n_{a}(s)$ (respectively, $n_{b}(s)$ ) denote the number of letters $a$ (respectively, $b$ ) in $s$. Put $d(s)=n_{a}(s)-n_{b}(s) \sqrt{2}, A=\{s \in S \mid d(s)>0\}, B=S \backslash A$. Consider the ideal $I$ generated in $S$ by all $s$ with $|d(s)|>3$. Factoring out the ideal $I$, we put $\bar{S}=S / I, \bar{A}=A \cup I / I, \bar{B}=B \cup I / I$. Let $\mathbb{R}$ be the ring of real numbers. Consider the contracted semigroup ring $\mathbb{R} \bar{S}$. Clearly, $\mathbb{R} \bar{S}=\mathbb{R} \bar{A}+\mathbb{R} \bar{B}$, as in [5].

Take any element $0 \neq x \in \mathbb{R} \bar{A}$, say $x=\sum_{i=1}^{m} r_{i} s_{i}$, where $r_{i} \in \mathbb{R}, 0 \neq s_{i} \in \bar{A}$. Denote by $N$ the ideal generated in $\mathbb{R} \bar{A}$ by $x$, and put $q=3 / \min _{i=1}^{m}\left|d\left(s_{i}\right)\right|$. Then it is routine to verify that $N^{q}=0$. Thus $\mathbb{R} \bar{A}$ is the sum of its nilpotent ideals. Similarly, $\mathbb{R} \bar{B}$ is a Wedderburn radical ring, too.

Let us inductively define a sequence of elements $t_{1}, t_{2}, \ldots \in\{a, b\}$. Put $t_{1}=a$. Suppose that $t_{1}, \ldots, t_{k}$ have been defined. Let $t_{k+1}=a$ if $d\left(t_{1} \cdots t_{k}\right)<0$, and let $t_{k+1}=b$ otherwise. An easy induction on $k$ shows that $-\sqrt{2}<d\left(t_{1} \cdots t_{k}\right)<1$, for all $k \geq 1$. Hence $t_{1} \cdots t_{k} \neq 0$ in $\mathbb{R} \bar{S}$. As in the second paragraph of the proof of the main theorem of [5], it follows that $a+b$ is not nilpotent in $\mathbb{R} \bar{S}$, because $t_{1} \cdots t_{k}$ is a summand of $(a+b)^{k}$. Thus $\mathbb{R} \bar{S}$ is not nil.

By the classical theorem of Amitsur the Jacobson radical of every finitely generated algebra over a nondenumerable field is nil. Hence $\mathcal{J}(\mathbb{R} \bar{S}) \neq \mathbb{R} \bar{S}$. Therefore there exists an ideal $P$ of $\mathbb{R} \bar{S}$ such that $\mathbb{R} \bar{S} / P$ is a primitive ring. This completes the proof.

Theorem 1 answers negatively all questions asked in $[6, \S 2.4]$.

Note that the main result of [5] follows from our Theorem 1.

For any function $f:(A \cup B) \rightarrow \mathbb{N}$, where $\mathbb{N}$ is the set of all natural numbers, let $I_{f}$ be the ideal generated in $S$ by all products $s_{1} \cdots s_{k}$ such that $k>$ $\max \left\{1, f\left(s_{1}\right), \ldots, f\left(s_{k}\right)\right\}$ and either $\left\{s_{1}, \ldots, s_{k}\right\} \subseteq A$ or $\left\{s_{1}, \ldots, s_{k}\right\} \subseteq B$. It is proved in [5] that, for any function $f$, the contracted semigroup ring $\mathbb{R}\left(S / I_{f}\right)$ is the sum of two locally nilpotent rings $\mathbb{R}\left(A / I_{f}\right)$ and $\mathbb{R}\left(B / I_{f}\right)$, and that there exists a function $g$ such that the ring $\mathbb{R}\left(S / I_{f}\right)$ is not nil for every function $f$ satisfying $f(s) \geq g(s)$ for all $s \in A \cup B$.

If we take $f(s)=\max \{g(s), 3 /|d(s)|\}$, for all $s \in A \cup B$, then clearly $I_{f} \subseteq I$, where $I$ is the ideal used in the proof of Theorem 1 . Thus the ring $\mathbb{R}(S / I)$ from the proof of our Theorem 1 is a homomorphic image of the ring $\mathbb{R}\left(S / I_{f}\right)$ introduced in [5].

\section{REFERENCES}

1. M. Ferrero and E. R. Puczyłowski, On rings which are sums of two subrings, Arch. Math. 53 (1989), 4-10. MR 90f: 16030

2. I. N. Herstein and L. W. Small, Nil rings satisfying certain chain conditions, Can. J. Math. 16 (1964), 771-776.

3. O. H. Kegel, Zur Nilpotenz gewisser assoziativer Ringe, Math. Ann. 149 (1963), 258-260. MR 28:3049

4. O. H. Kegel, On rings that are sums of two subrings, J. Algebra 1 (1964), 103-109. MR 29:3495

5. A. V. Kelarev, A sum of two locally nilpotent rings may be not nil, Arch. Math. 60 (1993), 431-435. MR 94c: 16025 
6. E. R. Puczyłowski, Some questions concerning radicals of associative rings, "Theory of Radicals", Szekszárd, 1991, Coll. Math. Soc. János Bolyai 61(1993), 209-227. MR 94j:16033

7. A. Salwa, Rings that are sums of two locally nilpotent subrings, Comm. Algebra 24 (1996), 3921-3931. CMP 97:01

Department of Mathematics, University of Tasmania, G.P.O. Box 252 C, Hobart, Tasmania 7001, Australia

E-mail address: kelarev@hilbert.maths.utas.edu.au 